\title{
Probing the 3d Spin Momentum with X-ray Emission Spectroscopy: The Case of Molecular-Spin Transitions
}

\author{
György Vankó,*,† Thomas Neisius, ${ }^{\ddagger}$ Gábor Molnár, ${ }^{\S}$ Franz Renz,, Szilvia Kárpáti, ${ }^{\perp}$ \\ Abhay Shukla," and Frank M. F. de Groot ${ }^{\circ}$ \\ European Synchrotron Radiation Facility, BP 220 F-38043 Grenoble Cedex, France, CP2M, Faculté des \\ Sciences et Technique, Université Paul Cézanne (Aix-Marseille III), 13397 Marseille Cedex 20, France, \\ Laboratoire de Chimie de Coordination, Centre National de la Recherche Scientifique UPR-8241, 205 route de \\ Narbonne, F-31077 Toulouse, France, Institut für Anorganische Chemie und Analytische Chemie, Johannes \\ Gutenberg-Universität Mainz, Duesbergweg 10-14, D-55099 Mainz, Germany, Department of Nuclear \\ Chemistry, Eötvös Loránd University, Pázmány sétány 1/A, H-1118 Budapest, Hungary, Institut de Minéralogie \\ et de Physique des Milieux Condensées, Université Pierre et Marie Curie, 4 place Jussieu, F-75252 Paris \\ Cedex 05, France, and Department of Inorganic Chemistry and Catalysis, Utrecht University, \\ Sorbonnelaan 16, 3584 CA Utrecht, Netherlands
}

Received: March 14, 2006; In Final Form: April 24, 2006

\begin{abstract}
We report X-ray emission spectra of $\mathrm{Fe}^{\mathrm{III}}, \mathrm{Fe}^{\mathrm{II}}$, and $\mathrm{Co}^{\mathrm{II}}$ spin-crossover compounds in their high-spin and low-spin forms. It is shown that all X-ray emission features are sensitive to the spin state. Variations of the $\mathrm{K} \beta$ and the $\mathrm{K} \alpha$ emission line shapes, which are in agreement with theory, can be used as quantitative probes of the spin state; it is suggested that with appropriate reference experiments one can extract the spin momentum for a general case. Resonant X-ray emission spectra unveil details of the redistribution of electrons on the $3 \mathrm{~d}$ levels associated with the spin-state change by revealing features at the X-ray absorption preedge not accessible through standard absorption measurements.
\end{abstract}

\section{Introduction}

Making use of isolated molecules (or their small assemblies) in electronic devices is expected to bring major advances to information technology. Molecules that exhibit bistability at around room temperature, for example, have potential applications in data storage and display devices. Promising candidates include the so-called spin-crossover complexes, which are coordination compounds of transition-metal ions with medium ligand field strength and an electronic configuration between $3 d^{4}$ and $3 d^{7}$. In these systems, the spin state of the molecules can be switched back and forth between the low-spin (LS) and the high-spin (HS) states by triggering a redistribution of the $3 \mathrm{~d}$ electrons on the $\mathrm{t}_{2 \mathrm{~g}}$ and $\mathrm{e}_{\mathrm{g}}$ orbitals with variation in the temperature or pressure, as well as irradiation with light or application of a high magnetic field. ${ }^{1,2}$ Recent research trends on such materials include systems containing two or more different metal sites ${ }^{3}$ and their behavior under extreme conditions such as high pressure ${ }^{4}$ with such experiments, challenges are faced because of the possible presence of different metal ions and sample environments with limited access to standard techniques.

Although many experimental techniques can indicate a change of the spin state through variations in electronic, optical, vibrational, magnetic, or structural properties, few can probe the spin quantitatively; even the most often applied magnetic

* To whom correspondence should be addressed. E-mail: vanko@esrf.fr

European Synchrotron Radiation Facility.

† Université Paul Cézanne.

$\S$ Centre National de la Recherche Scientifique.

" Johannes Gutenberg-Universität.

$\perp$ Eötvös Loránd University.

\# Université Pierre et Marie Curie Paris.

$\circ$ Utrecht University. susceptibility and Mössbauer spectroscopy measurements have limitations. Magnetic susceptibility is sensitive to all magnetic ions present in the system, including impurities, and it is not always a simple task to obtain the paramagnetic contribution of the investigated transition-metal ion from the measured magnetization. While Mössbauer spectroscopy is not influenced directly by the spin state, it can serve as a genuine probe through its sensitivity to the (re)distribution of the $3 \mathrm{~d}$ electrons; unfortunately, in the compounds of interest, it is practically limited to iron. Spectroscopies based on absorption or scattering of visible or infrared light are limited by the absorption and morphologic changes of the sample at a transition. The success of Mössbauer spectroscopy is due to its elemental selectivity and the large penetration power. Elemental selectivity is a wellknown property of X-rays, thus X-ray spectroscopies are natural candidates for such measurements. Soft X-ray absorption spectroscopy complemented by multiplet calculations have been shown to be a very appropriate tool for determining the spin state, and it has been used to resolve spin-state related problems. ${ }^{5-7}$ However, soft X-ray techniques are incompatible with the environments that extreme conditions (high pressure, high temperature) require. Hard X-rays were less often used in this context, although they fulfill all criteria to provide the means to probe the spin state of the bulk. X-ray absorption studies mostly focused on the near-edge and extended structures. ${ }^{8,9}$ The preedge region, which should be the most sensitive to the spin state, did not receive much attention for it is very weak and usually poorly resolved. X-ray emission spectroscopy (XES), however, was used to follow spin transitions in a few cases. ${ }^{10-12}$

The first proposition that XES bears information on the spin was made long ago. In a study of the $\mathrm{K} \beta$ emission spectra of manganese compounds, a satellite was observed on the low 
energy side of the main $\mathrm{K} \beta_{1,3}$ emission line, whose intensity was found to roughly correlate with the number of unpaired electrons on the transition metal. This satellite, referred to as $\mathrm{K} \beta^{\prime}$, was suggested to originate from the exchange interaction between the $3 \mathrm{~d}$ electrons and the $3 \mathrm{p}$ core hole left in the final state of the emission process. ${ }^{13}$ Despite theoretical confirmation of this interpretation, ${ }^{14-17}$ the spin sensitivity of the $\mathrm{K} \beta$ spectra has truly begun to be exploited only by recent high-pressure studies. The observation of a pressure-induced HS to LS transition in $\mathrm{FeS}^{10}$ was followed by a few other similar works. These studies, however, were essentially confined to the qualitative monitoring of the variation of the spin state, because, although the $K \beta$ spectra directly reflects the spin state through the exchange interaction, there is no straightforward way to obtain the expectation value of the total-spin angular momentum from the spectra.

Besides the $\mathrm{K} \beta$ emission, which arises from a $3 \mathrm{p} \rightarrow 1 \mathrm{~s}$ transition, electrons can fill the 1 s core hole from the $2 \mathrm{p}$ or valence orbitals giving rise to the $K \alpha$ or valence emission spectra, respectively. It was found that the width of the $K \alpha_{1}$ line depends on the spin state ${ }^{18}$ however, deviations from this behavior were also observed. ${ }^{19}$ Nevertheless, a systematic theoretical work concluded that the K $\alpha$ spectra encompass spinpolarized information. ${ }^{17}$ Therefore, it might be rewarding to inspect all XES features for spin sensitivity. Moreover, XES can be taken with different excitation energies around the absorption edge. This approach, called resonant X-ray emission spectroscopy (RXES) or resonant inelastic X-ray scattering (RIXS), can resolve the detailed structure of the X-ray absorption near-edge spectroscopy (XANES) preedge and offers complementary information similar to what can be obtained from soft X-ray spectroscopy. ${ }^{20,21}$

In this paper we make an extensive study of the spin sensitivity of X-ray emission lines measured off and on resonance, following $1 \mathrm{~s}$ core excitation or ionization of transition-metal ions. ${ }^{20} \mathrm{We}$ performed a systematic study of known spin-crossover systems of (quasi)octahedral metal-ligand core, which cover almost the entire $\mathrm{d}^{4}-\mathrm{d}^{7}$ range, have unambiguous spin states, and show diverse spin transitions. Besides providing benchmarks, our goal was to explore the potential of these techniques to obtain quantitative results on the spin. The interpretation of the measured spectra is assisted by theoretical calculations based on the crystal-field multiplet approach.

\section{Experimental Section}

2.1. Sample Preparation and Characterization. The Fe $\mathrm{Fe}^{\mathrm{III}}$ complex (hereafter $\left.\mathbf{F e}^{\mathrm{III}}\right)$, $\left[\mathrm{Fe}^{\mathrm{III}} \mathrm{L}(\mathrm{Py})\right] \mathrm{BPh}_{4}$ (L = bis(3-salicylidene-aminopropyl)-amine, $\mathrm{Py}=$ pyridine, $\mathrm{Ph}=$ phenyl), was prepared as described in ref 22 . The $\mathrm{Fe}^{\mathrm{II}}$ complex, denoted as $\mathbf{F e}^{\mathrm{II}},\left[\mathrm{Fe}^{\mathrm{II}}(\text { phen })_{2}(\mathrm{NCS})_{2}\right]$ (phen $=1,10$-phenantroline), was prepared by the extraction method described in ref 23. Finally, the preparation and characterization of $\left[\mathrm{Co}^{\mathrm{II}}(\text { terpy })_{2}\right] \mathrm{ClO}_{4} \cdot{ }^{1} /{ }_{2} \mathrm{H}_{2} \mathrm{O}$ (terpy $=2,2^{\prime}: 6^{\prime}, 2^{\prime \prime}$-terpyridine), which will be referred to as $\mathbf{C o}^{\text {II }}$, is given in ref 24 . The samples were characterized by elemental analysis, and the iron samples were also characterized with Mössbauer spectroscopy. Variable-temperature magneticsusceptibility data were taken on all samples with a Faradaytype magnetometer equipped with a continuous-flow Oxford Instruments cryostat. At 80 and $300 \mathrm{~K}$, where X-ray spectra were taken, all materials were entirely converted to the LS or the HS state, respectively, with the exception of $\mathbf{F e} \mathbf{e}^{\mathrm{III}}$, where the conversion to HS was $60 \%$ at $300 \mathrm{~K}$.

2.2. X-ray Spectroscopy. The experiments were carried out at beamlines ID16 and ID26 of the European Synchrotron
Radiation Facility. The undulator radiation was monochromatized with a cryogenically cooled $\mathrm{Si}(111)$ double-crystal monochromator; for RXES a Si(220) monochromator was used. The $\mathrm{X}$-ray spectra were taken at a scattering angle of $90^{\circ}$ with a 1 $\mathrm{m}$ diameter Rowland-circle spectrometer arranged in the horizontal plane, equipped with the appropriate spherically bent analyzer crystal and a Peltier-cooled Si diode to analyze and detect the emitted radiation, respectively. Different analyzer crystals were chosen to obtain optimal energy resolution, typically $1.5 \mathrm{eV}$. Namely, $\mathrm{Si}(620)$ reflection was used for the cobalt $\mathrm{K} \beta, \mathrm{Si}(531)$ for the iron $\mathrm{K} \beta$ and cobalt $\mathrm{K} \alpha$, and $\mathrm{Si}(444)$ for the iron $\mathrm{K} \alpha$ spectra. The XES and RXES spectra were collected by scanning the Bragg angle of the analyzer around the maxima of the emission lines; the detector was also translated and rotated accordingly. PFY XANES spectra were taken on the maximum of the $K \alpha_{1}$ emission line by scanning the incident energy through the absorption edge; this latter technique was also utilized to check for sample degradation in the beam. The samples were mounted in a standard closed-cycle helium cryostat. As we preferred to ensure the same state and conditions of the sample for the different measurements, we chose to swap the spectrometer setups while keeping the cryostat at the same temperature. This procedure can introduce artificial shifts to the emission line, similarly to those caused by position changes along the beam in a spectrometer having a horizontal plane of dispersion. Therefore, for the presentation of the $\mathrm{K} \alpha$ and $\mathrm{K} \beta$ spectra we applied the often-used practice, ${ }^{25,26}$ and aligned the spectra on the main peak. This does not affect the conclusions of the paper on the line-shape changes, whereas it allows better comparison of the HS/LS line shapes.

2.3. Multiplet Calculations. The $\mathrm{K} \alpha, \mathrm{K} \beta$, and $\mathrm{Fe} \mathrm{K}$ preedge spectra were modeled using the crystal-field multiplet theory. ${ }^{20}$ This approach includes the electrostatic (Coulomb) interactions, the spin-orbit coupling, and the crystal-field effects on every open shell. The spectrum is calculated from the sum of all possible transitions for an electron decaying from the $2 p(K \alpha)$ or $3 \mathrm{p}(\mathrm{K} \beta)$ level into the $1 \mathrm{~s}$ core hole. The off-resonance calculation assumes that the 1 s core hole does not affect the remaining electrons and calculates the decay from $1 \mathrm{~s}^{1} 3 \mathrm{~d}^{N}$ to $2 \mathrm{p}^{5} 3 \mathrm{~d}^{N}$ (or $3 \mathrm{p}^{5} 3 \mathrm{~d}^{N}$ ), where $N$ is given by the formal valence of the transition metal, for example, $N$ is equal to 5 for $\mathbf{F e}^{\mathrm{III}}$, etc. ${ }^{17}$ Quadrupolar $3 \mathrm{~d}^{N}$ to $1 \mathrm{~s}^{1} 3 \mathrm{~d}^{N+1}$ excitations were considered to simulate the preedge structure of $\mathbf{F e}^{\mathrm{II}}$.

\section{Results and Discussion}

3.1. Spin Sensitivity of the Different Emission Features. The full nonresonant K-emission spectra of $\mathbf{F e}^{\mathrm{II}}$, one of the most studied spin-transition complexes, are displayed in Figure 1 for both spin states. As it is seen, not only the $\mathrm{K} \beta$, but all emission features undergo a relevant modification upon the change in the spin state. The $\mathrm{K} \beta$ spectrum, as expected from the introduction, shows an intensity drop in the $\mathrm{K} \beta^{\prime}$ satellite region when the spin is diminished. The variation of the $K \alpha$ line shape is more complex, but the difference is ample. This sensitivity of the $\mathrm{K} \alpha$ emission lines is not completely unexpected, as a previous theoretical study suggested their use to obtain spinpolarized information. ${ }^{17}$ Both of these line-shape variations suggest potential to probe the spin state and will be analyzed later in more detail together with those of $\mathbf{F e}^{\mathrm{III}}$ and $\mathbf{C o}^{\mathrm{II}}$.

The highest energy features, which emerge when valence electrons (mostly centered on the ligands) fill the 1s core hole, are also modified at the spin transition. The short metal-ligand bond lengths in the LS state result in a relatively sharp and intense $\mathrm{K} \beta_{2,5}$ line, which is related to the overlap with the $N 2 \mathrm{p}$ 


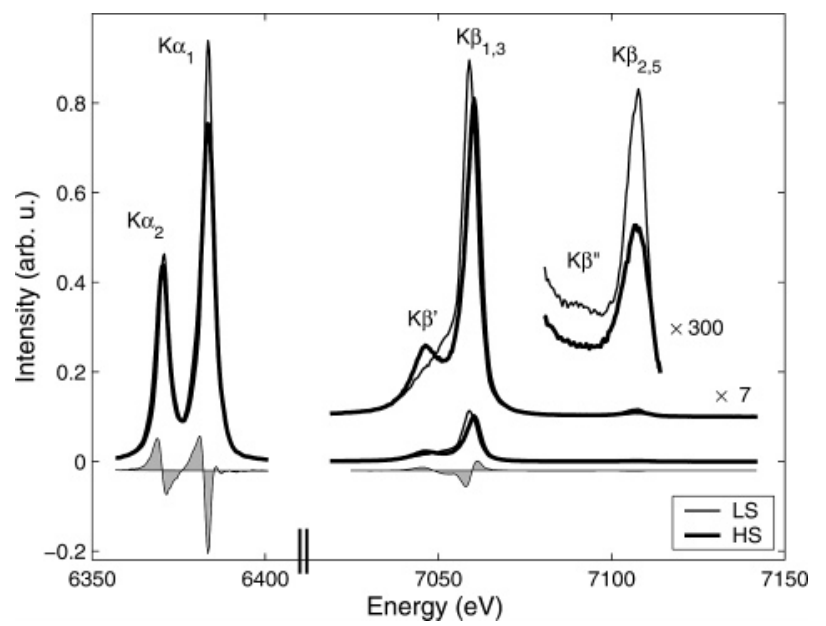

Figure 1. Full K-emission spectra of $\mathbf{F e}^{\mathrm{II}}$ in the HS (thick line) and LS (thin line) state. The $\mathrm{K} \beta$ and the valence emission regions are also shown in magnified forms. Below the spectra, the gray shaded area displays the HS-LS spectral difference.

orbitals. Even the $\mathrm{K} \beta^{\prime \prime}$ peak, related to transitions from the $N$ 2 s-dominated orbitals, is discernible. In the HS state all these features lose intensity because of the smaller overlaps caused by the $10 \%$ longer $\mathrm{Fe}-\mathrm{N}$ bond lengths. Hence, these modifications stem from the changes in the bonds, rather then directly from the redistribution of the $3 \mathrm{~d}$ electrons. Consequently, we must conclude that although this spectral region offers valuable information on the bonding, ${ }^{27}$ being weak and unrelated to the spin, it does not have prospects as probe of the spin state.

So far we have only considered nonresonant XES. However, the incident energy can be tuned to the absorption threshold such that it is sufficient to promote the 1s electron to unoccupied orbitals. The RXES spectrum obtained this way extends and complements the information that can be attained from K-edge XANES as it has been demonstrated recently on high-spin iron oxides $^{28,29}$ and will be shown below.

The Fe K X-ray absorption edge of the $\mathbf{F e}^{\mathrm{II}}$ complex was found to show major variations at the spin transition. ${ }^{30,31}$ The changes of the main $(1 \mathrm{~s} \rightarrow 4 \mathrm{p})$ absorption spectra were so substantial because of the large change induced by the modification of the bond lengths and, consequently, the unoccupied electron levels, that the weak intensity preedge features did not receive much attention, despite their inherent relation to the $3 \mathrm{~d}$ orbitals. As in the first row transition metals, the spin state is determined by the distribution of the $3 \mathrm{~d}$ electrons, we focus our attention to this spectral region, which, at a centrosymmetric octahedral iron site, originates entirely from quadrupolar $1 \mathrm{~s} \rightarrow$ $3 \mathrm{~d}$ transitions. It was recognized that despite the small intensity and poor resolution, these tiny peaks contain a wealth of information. ${ }^{32}$

The preedge of the (conventional) XANES spectra of this complex, taken from the literature ${ }^{30}$ is shown on the top of Figure 2. It reflects what can be intuitively expected: for an octahedral $3 \mathrm{~d}^{6}$ system the lower-lying $\mathrm{t}_{2 \mathrm{~g}}$ orbitals are partly empty and thus available for excitations only in the HS case; therefore, excitations at lower energy are expected for the HS spectrum when it is compared to that of the LS. Multiplet calculations for $1 \mathrm{~s}^{2} 3 \mathrm{~d}^{6} \rightarrow 1 \mathrm{~s}^{1} 3 \mathrm{~d}^{7}$ transitions in an octahedral crystal field reveal the underlying structures, which are shown as bars in the middle of the figure. Namely, the excitations that determine the spectra are transitions from the ${ }^{5} \mathrm{~T}_{2 \mathrm{~g}}$ quintet ground state to the ${ }^{4} \mathrm{~T}_{1 \mathrm{~g}},{ }^{4} \mathrm{~T}_{2 \mathrm{~g}}$, and ${ }^{4} \mathrm{~T}_{1 \mathrm{~g}}$ quartet states for the HS, and from the ${ }^{1} \mathrm{~A}_{1 \mathrm{~g}}$ to the ${ }^{2} \mathrm{E}_{\mathrm{g}}$ doublet state for the LS form. The structure of the HS spectrum is not resolved in XANES, as it is

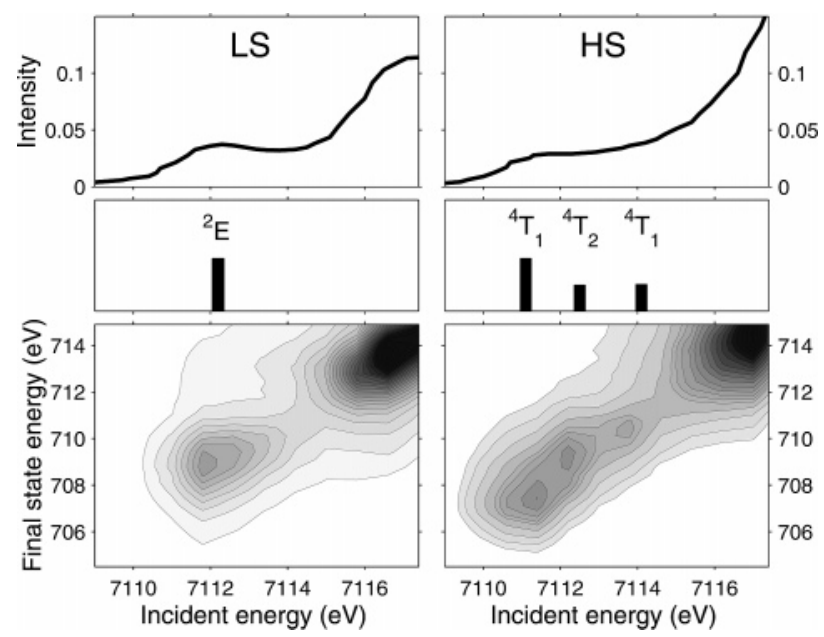

Figure 2. The preedge region of the Fe $\mathrm{K}$ absorption edge of $\mathbf{F e}^{\mathrm{II}}$. LS data is on the left; HS data is on the right. The figure shows XANES data from ref 30 (top), bars indicating the excitations calculated with crystal-field multiplet theory (middle), and $1 \mathrm{~s}^{2} \mathrm{p}_{3 / 2}$ resonant $\mathrm{X}$-ray emission spectra given as contour plots (bottom).

seen in the top of the figure, because of the short lifetime of the $1 \mathrm{~s}$ core hole leading to a natural line width of $1.3 \mathrm{eV} .^{33}$

Details of the underlying transitions can be unveiled using RXES, that is, combining absorption spectroscopy with emission; contrasting such experiments to theory opens a way to the simultaneous determination of the local structure, the valence and the spin states. ${ }^{21}$ This is indeed in the $1 \mathrm{~s} 2 \mathrm{p}_{3 / 2}$ RXES spectra shown in Figure 2. In agreement with theory, the HS spectrum reveals all three distinct features at incident energies of 7111.4, 7112.2 , and $7113.6 \mathrm{eV}$, while the LS spectrum consists of a single preedge feature at $7111.8 \mathrm{eV}$.

This result also implies that partial fluorescent yield (PFY) XANES spectra ${ }^{34}$ (also known as constant emission energy $\operatorname{scan}^{35,21}$ ), which corresponds to a diagonal sampling of the RXES surface, can serve as a useful tool to monitor the variations of the electronic configuration with considerably less experimental efforts than RXES but with better energy resolution and better separation of spectral features than conventional XANES. ${ }^{34,20}$ PFY XANES has its limitations, ${ }^{36}$ but usually retains most of the advantages of RXES and combines it with rapidity of the experiment. From the techniques discussed here, PFY XANES over the preedge region with the spectrometer tuned to the maximum of the most intense emission line, $\mathrm{K} \alpha_{1}$, provides the fastest way of following the spin transition and, in general, variations of the electronic structure, when the absorption on the incident beam is not excessive.

PFY XANES over the preedge region becomes even more relevant when the variation of the bond lengths and, accordingly, the variation of the unoccupied electron density of states are very small at spin transition, so the main edge structure is not much affected. This is the case for $\mathbf{C o}^{\mathrm{II}}$ and also for other spintransition compounds. ${ }^{31}$ Figure 3 displays the PFY XANES spectra of $\mathbf{C o}^{\mathrm{II}}$. No edge shift is observed, and the main XANES features show some intensity changes but no shifts in energy positions. The preedge region, by contrast, is altered significantly, and the remarkably resolved features give unmistakable indication of the electron redistribution on the $3 \mathrm{~d}$ orbitals. In such a case, the measurement limited to the preedge region is fast enough to follow transitions on the time scale of a minute.

3.2. Line-Shape Variations of $K \boldsymbol{\alpha}$ and $\mathbf{K} \boldsymbol{\beta}$. After having demonstrated that all XES features, nonresonant and resonant, vary with the spin state, we analyze the $\mathrm{K} \beta$ and the $\mathrm{K} \alpha$ emission lines for all complexes and compare them to results of our 


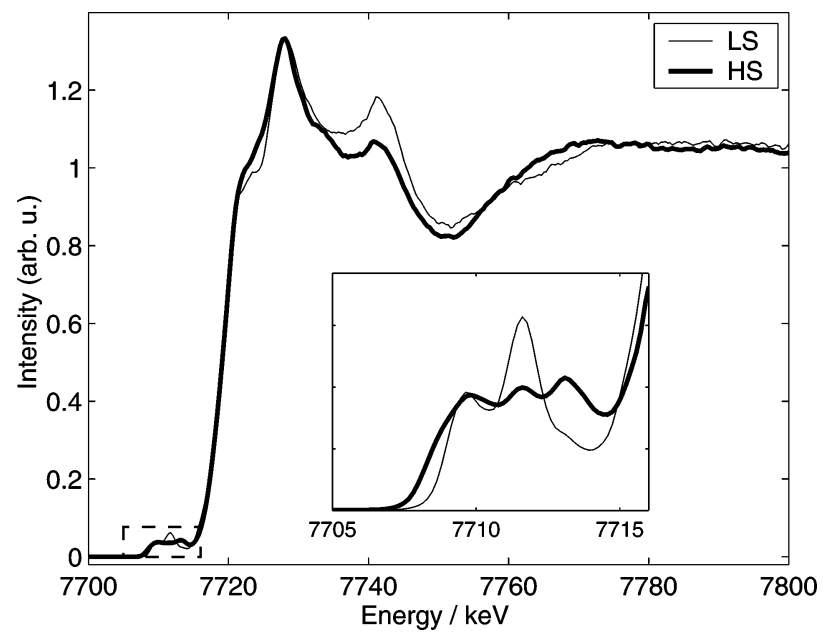

Figure 3. Partial fluorescence yield XANES spectra (taken on the $\mathrm{K} \alpha_{1}$ emission line) of $\mathbf{C o}^{\text {II }}$. The inset shows the preedge region.

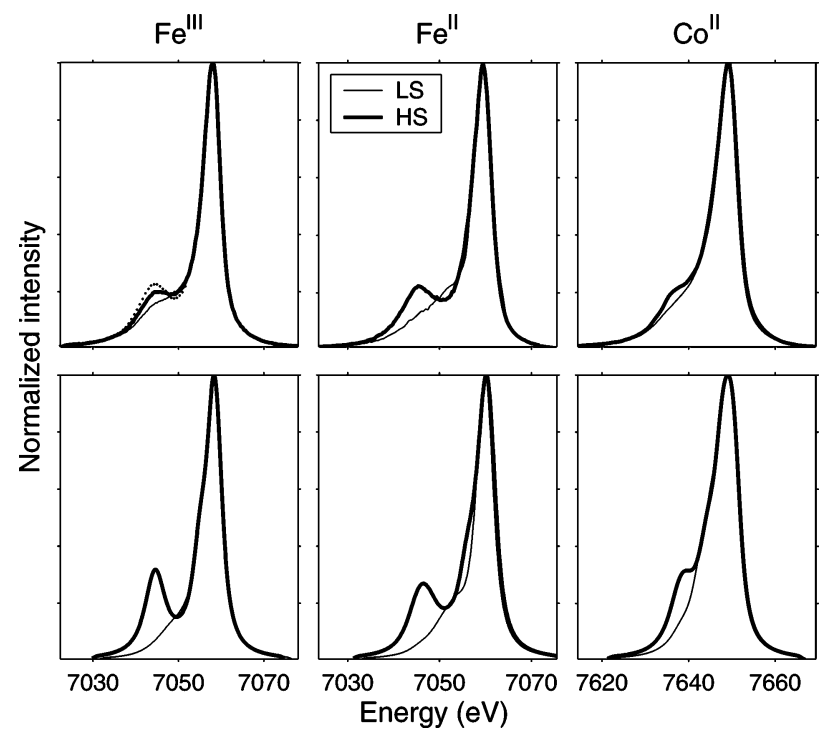

Figure 4. Measured (top) and calculated (bottom) $\mathrm{K} \beta$ spectra of the studied complexes. The spectra are normalized to the maximum for better visibility of the satellite region. Besides the experimental spectra of $\mathbf{F e}^{\mathrm{III}}$, a constructed one for $\gamma_{\mathrm{HS}}=1$ is shown as a dotted line.

theoretical study, the ultimate aim being quantitative determination of the spin state or spin momentum.

Figure 4 presents the $\mathrm{K} \beta$ spectra of the three studied compounds. As it is apparent from the figure, our results substantiate previous claims that the intensity of the $\mathrm{K} \beta^{\prime}$ satellite reflects the spin state differences. The spectra calculated with crystal-field multiplet theory (performed with different crystalfield splittings to obtain different spin states) are in good qualitative agreement with the measured spectra and satisfactorily reproduce the nature of the observed changes. (The $\mathrm{K} \beta^{\prime}$ peak heights are exaggerated in the calculations, which is a consequence of the term-dependent lifetime broadening that is not accounted for properly. ${ }^{16}$ ) The calculations also confirm that the $\mathrm{K} \beta^{\prime}$ region is practically completely spin-polarized, and the large separation of this feature from the main line is a consequence of the large $3 p-3 d$ exchange interaction of $15-$ $20 \mathrm{eV}$ in the final state. It is also clear from theory that the quantitative determination of the spin on the basis of the $\mathrm{XE}$ spectra is difficult even for the $\mathrm{K} \beta$ spectra because of the underlying complex multiplet term structure, which does not depend only on the spin state. This excludes comparison of ions in very different local geometries. On the other hand, it is

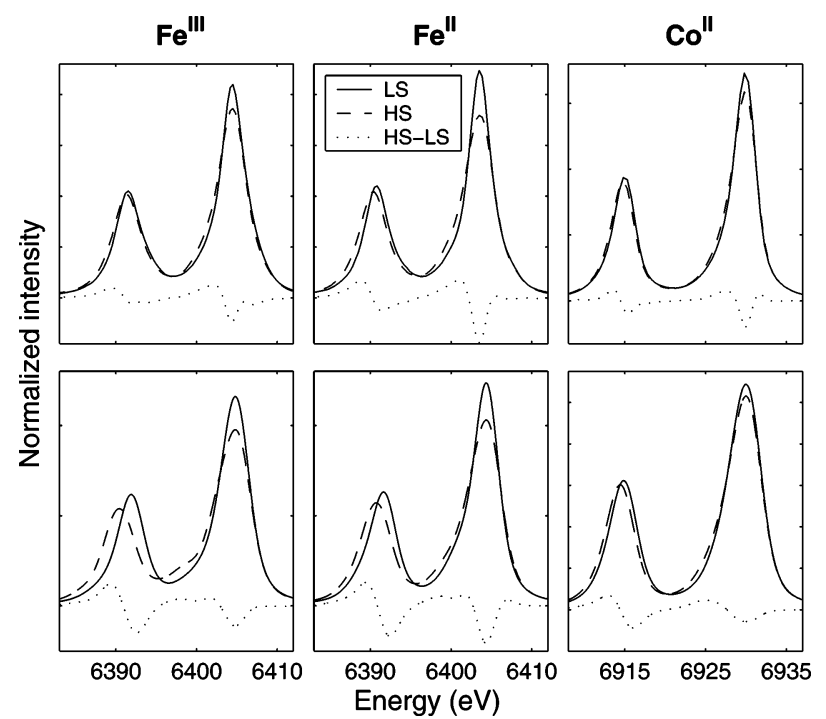

Figure 5. Measured (top) and calculated (bottom) Ka spectra of the studied complexes. Outer spectral regions (tails) have been omitted for clarity; the theoretical HS-LS difference spectrum of $\mathbf{F e}^{\mathrm{III}}$ has been scaled down by 0.6 to match the extent of conversion at the experiment.

remarkable that crystal-field theory, that is, an ionic picture provides such a good description of the spectral changes for these covalent compounds; this indicates that for locally similar atomic environments, similar line-shape changes can be expected upon variation of the spin state.

The alteration of the K $\alpha$ spectra with the spin state, displayed in Figure 5, cannot be unraveled that easily. What is apparent from the figure is that in the LS state the $K \alpha_{1}$ line (the peak at higher energy) is stronger and narrower than in the HS state. The spectral variations are not as striking as in the $\mathrm{L}_{2,3}$ soft $\mathrm{X}$-ray absorption spectrum, ${ }^{6}$ which is related to the K $\alpha \mathrm{XES}$ as it has also a $2 p$ core hole in the final state; the differences are due to the substantial lifetime broadening of the 1s core hole and the different transitions that lead to the formation of the same $2 p$ core hole. The branching ratio (the $2 p_{3 / 2}$ intensity fraction), which was found to change significantly at the L-edge, does not vary with the spin state in the K $\alpha$ XES. This is in agreement with theory and also follows from the difference in the transitions. The $\mathrm{K} \alpha_{1} / \mathrm{K} \alpha_{2}$ peak height ratios are nevertheless modified and can be easily determined and are listed below in the sequence material HS ratio/LS ratio: $\mathbf{F e}^{\mathrm{III}}, 2.01: 1.83$; $\mathbf{F e}^{\mathrm{II}}$, 2.03:1.72; $\mathbf{C o}^{\mathrm{II}}, 1.85: 1.74$. The $\mathrm{K} \alpha$ lines are broader at high spin: the information content of this will be discussed later.

The energy difference of the two lines is also affected, but only very slightly, since the large separation of the $K \alpha_{1}$ and $\mathrm{K} \alpha_{2}$ lines derives from the $2 \mathrm{p}$ spin-orbit interaction. The $2 \mathrm{p}-$ $3 \mathrm{~d}$ exchange interaction, which is responsible for the spin sensitivity of the $\mathrm{K} \alpha$ spectrum, is only $1-2 \mathrm{eV}$. Accordingly, we observe only moderate changes in intensity and broadening and very slight line shifts as seen in the HS-LS difference spectra. Multiplet calculations reproduce the experimental spectra reasonably well, which confirms that line-shape variations are due to the change in the spin state. (The smaller differences seen below the $K \alpha_{2}$ peaks in the experimental spectra only indicate that theory overestimates the $2 p-3 d$ exchange interaction, which leads to larger virtual shift of the $\mathrm{K} \alpha$ lines.) Though line-shape variations are not as striking as in the $\mathrm{K} \beta$ spectra, the overall spectral variation is quite obvious through the difference and, as it was shown in Figure 1, in terms of counts, the observable change in the $\mathrm{K} \alpha$ spectrum is 1 order of magnitude larger than in case of $\mathrm{K} \beta$. Consequently, significantly 


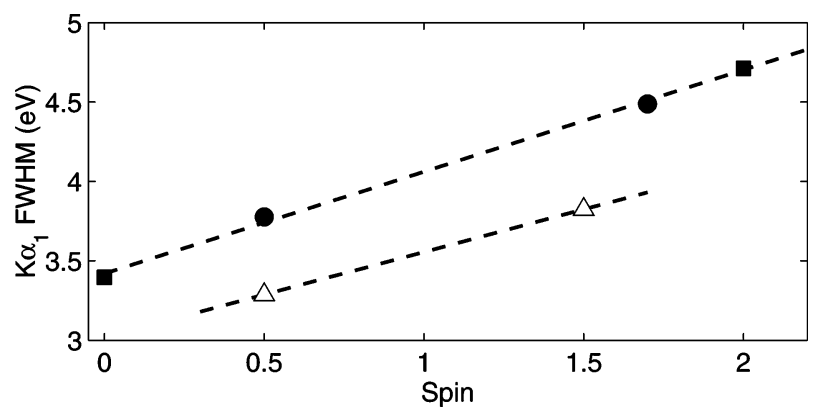

Figure 6. Measured $\mathrm{K}_{1}$ line widths in the different spin states. The spectral widths of $\mathbf{F e}^{\mathrm{III}}, \mathbf{F e}^{\mathrm{II}}$, and $\mathbf{C o}^{\mathrm{II}}$ are represented by circles, squares, and triangles, respectively. (Note that for the spin value of "HS" $\mathbf{F e}^{\mathrm{III}}$, we took into account the uncomplete spin conversion.) The dashed lines are guides to the eye.

higher signal-to-noise ratio can be achieved with the K $\alpha$ spectra; this makes it a very promising tool to probe the spin state.

3.3. Determination of the Spin Momentum from XES. The task of extracting quantitative information from XE spectra is difficult because the measured envelope is due to a complex multiplet structure resulting in a multitude of transitions of varying line widths. Several theoretical propositions and practical approaches exist, however, which make an attempt at relating the XES lines to the $3 \mathrm{~d}$ spin momentum. In what follows, we make a brief survey of them and test their usability.

It was proposed that characteristic energy splittings in the $\mathrm{XE}$ spectra are proportional to the spin momentum of the $3 \mathrm{~d}$ electrons, $S$, according to the equation

$$
\Delta E=J(2 S+1)
$$

where $J$ stands for the $3 \mathrm{~d}$-core hole exchange interaction energy. ${ }^{13,19}$

In the case of $K \alpha$, multiplet theory suggests that eq 1 concerns the width of the $K \alpha_{1}$ line. ${ }^{18,19,21}$ To our knowledge, this has not been exploited yet in following spin transitions, the plausible reason being the observed deviations from this expected behavior, for example, in HS compounds of transitional metals. ${ }^{19,37}$ However, as it can be seen in Figure 6, for our model compounds, these line widths clearly correlate with the spin, which allows us to distinguish different spin states. The widths of $\mathrm{K} \alpha_{1}$ in the Fe complexes show a remarkable behavior, since regardless of the differences in the valence state, they vary linearly with the spin. The spectral width of $\mathbf{H S} \mathbf{F e}^{\mathrm{III}}$ (which has a mixed spectrum with $\gamma_{\mathrm{HS}}=60 \%$ corresponding to $\bar{S}=$ 1.7) falls exactly on the line made up by those of the other spectra with "clean" spin states; this indicates applicability to both classifying spin states and following transitions quantitatively. All in all, this suggests a great potential to quantitative probing of the spin momentum. It cannot be expected, however, that the same relation will hold for any atomic environment. For instance, using the relationship between line widths and spin presented in Figure 6 we could derive $S \approx 1$ for the case of Fe metal; similar efforts failed also in the case of highly correlated oxides. ${ }^{19,37}$ Nevertheless, the present and earlier data ${ }^{19}$ suggest that the linear relation between the $K \alpha_{1}$ width and the total-spin momentum holds for coordination compounds.

In the case of $\mathrm{K} \beta$, eq 1 is expected to describe the energy separation of the $\mathrm{K} \beta_{1,3}$ main peak and the $\mathrm{K} \beta^{\prime}$ satellite. ${ }^{13}$ However, the large broadening and the small intensity of the satellite make the practical use of the above equation unfeasible at small numbers of unpaired $3 \mathrm{~d}$ electrons. Alternatively, one can obtain similar information by using the energy difference between the center of mass of the spectrum and the main peak: this very easily computable quantity, which we will denote as $\Delta E_{\mathrm{CP}}$, should be a comparable measure of the spin momentum.

For $\mathrm{K} \beta$, as it was shown in Figure 4, the satellite intensity is increased at higher spin, which should also make possible the estimation of the spin momentum. It was proposed on the basis of theoretical considerations that the $\mathrm{K} \beta^{\prime}$ intensity should be $S / S+1$ times that of the $\mathrm{K} \beta_{1,3}$. Experimentally only the trend was confirmed; the calculated values were found significantly overestimated. ${ }^{13}$ Nevertheless, this is the most often used method to determine the variation of the spin from $\mathrm{K} \beta \mathrm{XES}$; it is usually performed by scaling the satellite intensities of the extreme spectra to known or expected $S$ values. ${ }^{26,38}$

Suitable reference spectra for the different possible spin states can also be used by fitting their linear combination to the experimental spectrum. However, adequate reference spectra for each possible spin state with the closest possible atomic arrangement, bonding, and experimental conditions to those of the studied metal ion cannot always be had. In certain cases statistical quality of the spectra does not permit this approach. Nevertheless, the iron spectra presented here were successfully used to reproduce the $\mathrm{K} \beta$ spectra for pressure-induced spin state changes of iron in $\mathrm{Mg}_{0.9} \mathrm{Fe}_{0.1} \mathrm{SiO}_{3}$, through model functions constructed by superposing the spectra in proportion to the site occupations and valence distributions. ${ }^{39}$ The modeled and the measured spectra of this mineral showed good agreement. This also illustrates that XES of ions with similar coordination geometry can be similar, despite the big differences in the ligands' electronic (spectrochemical) properties. In other words, crystal-field theory works well in describing these line shapes.

The last approach we mention is to follow the variation of the spin state through the integrals of the absolute values of the difference spectra (hereafter IAD). ${ }^{25,40}$

Let us note the HS and LS XE spectral functions as $h(E)$ and $l(E)$, respectively, which are normalized to unit area at integration. Using them, the IAD value for the complete spin transition can be given as $\mathrm{IAD}_{\mathrm{HL}}=\int|h(E)-l(E)| \mathrm{d} E$. A spectrum in the transition region is a superposition of those of the two spin states, thus it can be expressed as $s=\gamma_{\mathrm{HS}} h+(1$ $\left.-\gamma_{\mathrm{HS}}\right) l$, where $\gamma_{\mathrm{HS}}$ is the high-spin fraction. Its difference from the low-spin reference $l$ is $s-l=\gamma_{\mathrm{HS}}(h-l)$. The integral of its absolute value is

$$
\operatorname{IAD}(s)=\int|(s(E)-l(E))| \mathrm{d} E=\gamma_{\mathrm{HS}} \mathrm{IAD}_{\mathrm{HL}} .
$$

Consequently, being proportional to $\gamma_{\mathrm{HS}}$, the IAD value is indeed a good measure of the extent of the transformation. The reference used can obviously be the HS spectrum as well or more generally any linear combination of the two spectra.

The advantages of this approach are numerous. It uses the information of the full spectrum, not only a part of it: as it is seen in the difference spectra in Figure 1, the spectral shape variations extend over a range of about $30-35 \mathrm{eV}$. Notably, it works for the $K \alpha$ spectra as well, since the above relations are valid for any spectral functions whose area are normalized to unity. It is model independent, it can be performed without invoking results of theoretical calculations, it relies only on IAD values from reference spectra, and the raw data need only a simple preparation before the analysis can be used, so it is a promising tool for the rapid determination of the spin state from the XE spectra.

The extent of the IAD variations over a unit change in the spin are similar for the studied compounds. The possession of such $\mathrm{IAD}_{\mathrm{HL}} / \Delta S$ relations might lead to spin-momentum determination from XES using a single reference spectrum or to 
estimate the spin variation between two experimental points without the use of any references. Moreover, there are indications that the approach can be extended to more than two spin states. In a recent study on cobaltates, the IADs were found varying linearly with the spin. ${ }^{40}$ These observations suggest that the IAD has the potential to extend the quantitative spin momentum determination to general cases.

3.4. Test of the Quantitative Approaches. The suitability of the above-discussed quantitative approaches were tested on a set of simulated $K \beta$ spectra. As seen in Figure 4, the line shapes and their variations can have stark differences in terms of separation of the satellite from the main line as well as satellite intensity depending on the investigated ion. Therefore, two sets of spectra, $\mathbf{F e}^{\mathrm{II}}$ with a distinct satellite and $\mathbf{C o}^{\mathrm{II}}$ with unresolved satellite, were constructed as superpositions of the pure LS and HS spectra to obtain $\gamma_{\mathrm{HS}}=0,0.1,0.2, \ldots, 1$. Since experimental data measured on $\mathbf{F e}^{\mathrm{II}}$ under favorable conditions proved that the center of mass (COM) is constant within the limits of experimental error, the HS and LS reference spectra were aligned to their COM; such a procedure has also been suggested by Glatzel. ${ }^{21}$ Before creating the intermediate spectra, the reference ones were also resampled to an energy step size of $0.02 \mathrm{eV}$ (with the application of quadratic spline) to avoid numerical problems and instabilities, and they were normalized to the spectral area. From each set of spectra, another has also been constructed to imitate realistic, statistically limited experimental data by resampling the spectra for a $0.2 \mathrm{eV}$ step size, and generating random deviations of normal distribution so that the results correspond to counting statistics of spectra with 50000 counts at their peak.

As we saw above, the different quantitative approaches are based on energy shifts or intensity variations. The energy shifts are measured by the $\Delta E_{\mathrm{CP}}$ (the energy difference between the main peak and the COM), whose use here is essentially equivalent to the use of the energy shift of the main line, an approach also applied sometimes at experiments. ${ }^{39,41}$ Besides, $\Delta E_{\mathrm{CM} 1}$ is calculated similarly, but for the latter the first moment of the upper half of the $\mathrm{K} \beta_{1,3}$ line is taken for peak position as suggested in ref 21 .

The intensity changes of the $\mathrm{K} \beta^{\prime}$ satellite were calculated as the intensity difference from the LS integrated up to the isobestic point of the spectra (around 7047 and $7640 \mathrm{eV}$ for $\mathbf{F e}^{\mathrm{II}}$ and $\mathrm{Co}^{\mathrm{II}}$, respectively). These are denoted as $I\left(\mathrm{~K} \beta^{\prime}\right)_{\mathrm{Ca}}, I\left(\mathrm{~K} \beta^{\prime}\right)_{\mathrm{Cm}}$, $I\left(\mathrm{~K} \beta^{\prime}\right)_{\mathrm{Pa}}$, and $I\left(\mathrm{~K} \beta^{\prime}\right)_{\mathrm{Pm}}$, the letters of the subscript stand for the different alignments and different normalizations that were used in the analysis of the simulated spectra. Namely, the capital subscript stands for the spectral shift: "P" for main peak and "C" for COM, while "a" and "m" denotes the normalization of the spectra to the area, and to the maximum intensity, respectively. Finally, the IAD approach was also tested with two different alignments of the spectra.

Figure 7 summarizes how the $\gamma_{\mathrm{HS}}$ calculated with a given approach differs from the "real" one, that is, the one used at the simulation. In general, it is apparent that practically all approaches give values which correlate with the spin, thus allowing qualitative monitoring of the spin momentum variation or classifying spin states. Most of these approaches, however, are not adequate to probe the transition precisely.

The IAD with aligning the spectra on the peak $\left(\mathrm{IAD}_{\mathrm{P}}\right)$ did not give very satisfying results, but aligning on the $\mathrm{COM}\left(\mathrm{IAD}_{\mathrm{C}}\right)$ excellently reproduces the high-spin fractions, as expected on the basis of eq 2 . Besides $\mathrm{IAD}_{\mathrm{C}}, I\left(\mathrm{~K} \beta^{\prime}\right)_{\mathrm{Ca}}$ gives also a perfect linear variation with $\gamma_{\mathrm{HS}}$. The other approaches are all subject to systematic errors of different extent as reported in the figure.

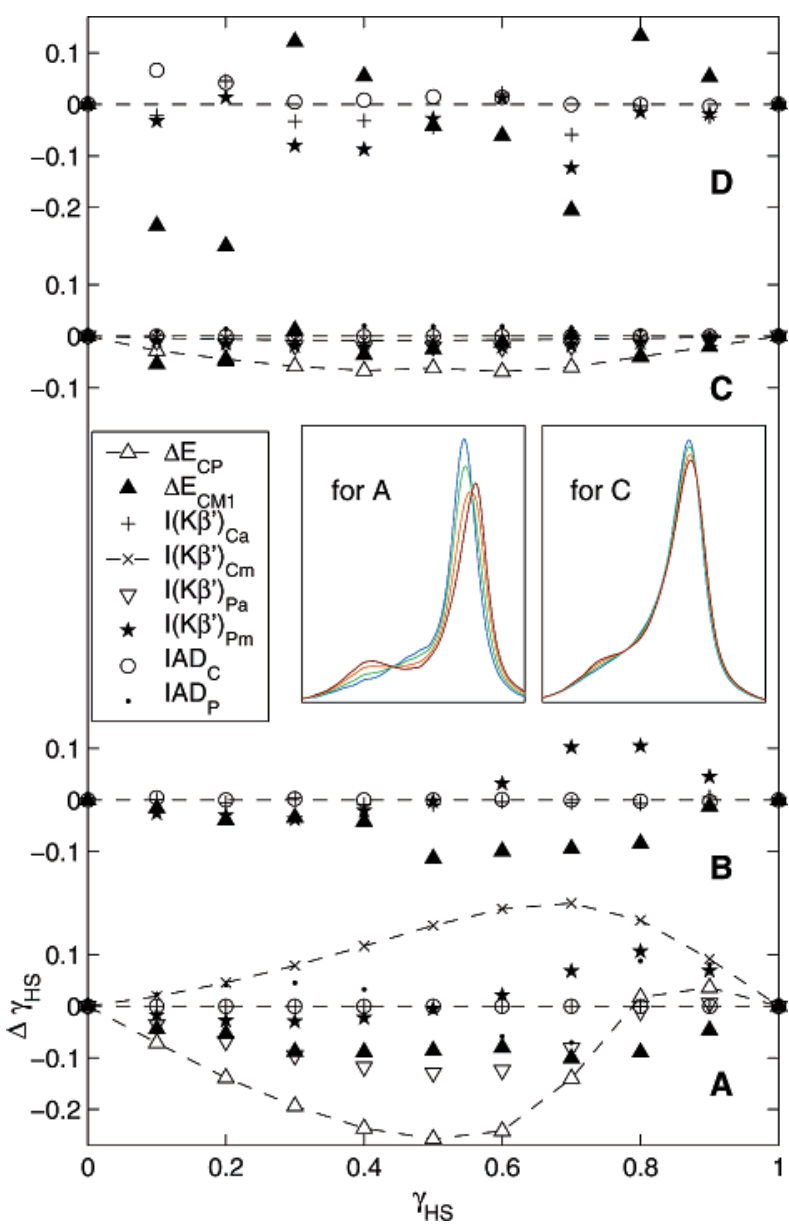

Figure 7. Deviations of the determined $\gamma_{\mathrm{HS}}$ values obtained on simulated spectra. For the notation of the different approaches consult the text. Parts A and B show values obtained on the spectra constructed from those of the $\mathbf{F e}^{\mathrm{II}}$ (case of a distinct $\mathrm{K} \beta^{\prime}$ ) without and with statistical errors, respectively. For parts $\mathrm{C}$ and $\mathrm{D}$, spectra of $\mathbf{C o}^{\mathrm{II}}$ (case of a shoulder-like $\mathrm{K} \beta^{\prime}$ ) were used. For simplicity, in parts B and D only the most successful and the most applied approaches are retained. Two insets in the middle of the figure illustrate the simulated spectra.

The largest deviations were met when the energy shifts were applied. Two of the approaches that concern satellite intensities systematically over- or underestimate the extent of the transition $\left(I\left(\mathrm{~K} \beta^{\prime}\right)_{\mathrm{Cm}}\right.$ and $\left.I\left(\mathrm{~K} \beta^{\prime}\right)_{\mathrm{Pa}}\right)$. It is interesting to examine the case of $I\left(\mathrm{~K} \beta^{\prime}\right)_{\mathrm{Pm}}$, that is the integrated satellite intensity of spectra aligned to the main peak and normalized to the peak maxima. This commonly used approach is conceptually wrong, as the satellite intensity is artificially scaled up by the normalization. Interestingly, this does not cause the obtained values to be systematically too high or too low over the full region. Thus the error on the satellite intensity has an interplay with other errors that stem from the simplification of the spectral changes to the variation of the satellite intensity, which can in certain cases compensate. Finally, the success of $I\left(\mathrm{~K} \beta^{\prime}\right)_{\mathrm{Ca}}$ can be understood as it is obtained similarly to the $\mathrm{IAD}_{\mathrm{C}}$, only the integration range is smaller. The above conclusions hold also for moderate quality spectra if the satellite is distinct (e.g., in the case of $\mathbf{F e}^{\mathrm{II}}$ ), as seen in Figure 7B. However, the situation is completely different in the case of spectra with a small and unresolved satellite (case of $\mathbf{C o}^{\mathrm{II}}$ ). For optimal spectra, all of these approaches provide a reasonably good description of the extent of the transition, cf. Figure 7C. Applying similar statistical conditions as to the first set of spectra in $\mathrm{B}$, however, introduces big instabilities and large deviations (Figure 7D). The best performance is again obtained with the $\mathrm{IAD}_{\mathrm{C}}$ and $I\left(\mathrm{~K} \beta^{\prime}\right)_{\mathrm{Ca}}$. From 
these, we expect that the $\mathrm{IAD}_{\mathrm{C}}$ approach is more robust (less sensitive to statistical limitations of the data) in general, as the larger spectral range used results in quantities at integration that are an order of magnitude greater.

\section{Conclusions}

We show that hard X-ray spectroscopies provide sensitivity to the spin state combined with unique qualities: element selectivity, bulk sensitivity, compatibility with extreme conditions, and relatively straightforward experimental conditions. This is illustrated with spin-state studies using partial fluorescence yield XANES and resonant X-ray emission at the preedge which is directly related to the electron distribution on the $3 \mathrm{~d}$ orbitals. PFY XANES offers rapidity, while RXES offers more detailed information regarding the spin state, valence state, and local geometry.

We also show that XES with careful line-shape analysis is on the way to becoming an established technique to probe the spin state. Combined with the powerful beams of third generation synchrotron radiation sources, XES can even be applied when the concentration of the studied metal ion or the amount of sample is very small, despite the possible presence of large amounts of other magnetic ions. Moreover, XES offers to work with sample environments with high absorption at the $\mathrm{K}$ edges of transition metals, as the energy of the ionizing radiation can be increased to minimize the absorption loss of the incident beam on the way to the sample. $\mathrm{K} \beta$ spectra offer an easy way to follow qualitatively the variation of the spin through the variation of the $\mathrm{K} \beta^{\prime}$ satellite intensity, while the higher fluorescence yield $\mathrm{K} \alpha$ spectra provide larger overall line-shape changes. In addition, the latter offer a second tool to probe the spin state via the variation of the line width. Finally we show how the variation of the total-spin momentum may be monitored quantitatively and discuss the determination of the total-spin momentum for general cases.

Acknowledgment. We are indebted to Dr. C. Cartier dit Moulin for sending us his XANES data on Fe Fe $^{\mathrm{II}}$ F.R. was supported by the Johannes Gutenberg-Universität Mainz (MWFZ and Forschungsfond) and the DFG-SPP 1137 (Grant RE1627/ 1-3). This paper is dedicated to Prof. Attila Vértes on the occasion of his $70^{\text {th }}$ birthday.

\section{References and Notes}

(1) Gütlich, P.; Hauser, A.; Spiering, H. Angew. Chem., Int. Ed., Engl. 1994, 33, 2024-2054.

(2) Gütlich, P., Goodwin, H. A., Eds.; Spin Crossover in Transition Metal Compounds I-III. in Topics in Current Chemistry; SpringerVerlag: Berlin-Heidelberg, Germany, 2004; Vols. 233-235.

(3) Kahn, O. Nature 1999, 399, 21-22.

(4) Ksenofontov, V.; Gaspar, A. B.; Gütlich, P. Top. Curr. Chem. 2004, $235,23-64$.

(5) van der Laan, G.; Thole, B. T.; Sawatzky, G. A.; Verdaguer, M. Phys. Rev. B 1988, 37, 6587-6589.

(6) Cartier dit Moulin, C.; Rudolf, P.; Flank, A. M.; Chen, C. T. J. Phys. Chem. 1992, 96, 6196-6198.

(7) Lee, J.-J.; Sheu, H.-S.; Lee, C.-R.; Chen, J.-M.; Lee, J.-F.; Wang, C.-C.; Huang, C.-H.; Wang, Y. J. Am. Chem. Soc. 2000, 122, 5742-5747.

(8) Briois, V.; Sainctavit, P.; Long, G.; Grandjean, F. Inorg. Chem. 2001, 40, 912-918.
(9) Hannay, C.; Hubin-Franskin, M.-J.; Grandjean, F.; Briois, V.; Itié, J.-P.; Polian, A.; Trofimenko, S.; Long, G. J. Inorg. Chem. 1997, 36, 55805588.

(10) Rueff, J.-P.; Kao, C.-C.; Struzhkin, V. V.; Badro, J.; Shu, J.; Hemley, R. J.; Mao, H. K. Phys. Rev. Lett. 1999, 82, 3284-3287.

(11) Vankó, G.; Neisius, T.; Renz, F.; Kárpáti, S.; Shukla, A.; Mirone, A.; de Groot, F. ESRF Highlights 2002, 59-60.

(12) Rueff, J.-P.; Mattila, A.; Badro, J.; Vankó, G.; Shukla, A. J. Phys. Condens. Matter 2005, 17, S717-S726.

(13) Tsutsumi, K.; Nakamori, H.; Ichikawa, K. Phys. Rev. B: Condens. Matter Mater. Phys. 1976, 13, 929-933.

(14) Peng, G.; de Groot, F.; Hämäläinen, K.; Moore, J.; Wang, X.; Grush, M.; Hastings, J.; Siddons, D.; Amstrong, W.; Mullins, O.; Cramer, S. J. Am. Chem. Soc. 1994, 116, 2914-2920.

(15) de Groot, F. M. F.; Pizzini, S.; Fontaine, A.; Hämäläinen, K.; Kao, C. C.; Hastings, J. B. Phys. Rev. B: Condens. Matter Mater. Phys. 1995, $51,1045-1052$

(16) Taguchi, M.; Uozumi, T.; Kotani, A. J. Phys. Soc. Jpn. 1997, 66, $247-256$.

(17) Wang, X.; de Groot, F. M. F.; Cramer, S. P. Phys. Rev. B: Condens. Matter Mater. Phys. 1997, 56, 4553.

(18) Meisel, A.; Leonhardt, G.; Szargan, R. X-ray Spectra and Chemical Binding; Spinger Series in Chemical Physics, Vol. 37; Springer-Verlag: Berlin, Germany, 1989.

(19) Kawai, J.; Suzuki, C.; Adachi, H. Konishi, T.; Gohshi, Y. Phys. Rev. B: Condens. Matter Mater. Phys. 1994, 50, 11347-11354.

(20) de Groot, F. M. F. Chem. Rev. 2001, 101, 1779-1808.

(21) Glatzel, P.; Bergmann, U. Coord. Chem. Rev. 2005, 249, 65-95.

(22) Matsumoto, N.; Ohta, S.; Yoshimura, C.; Ohyoshi, A.; Kohata, S.;

Okawa, H.; Maeda, Y. J. Chem. Soc., Dalton Trans. 1985, 2575-2584.

(23) König, E.; Madeja, K. Inorg. Chem. 1967, 6, 48-55.

(24) Oshio, H.; Spiering, H.; Ksenofontov, V.; Renz, F.; Gütlich, P. Inorg. Chem. 2001, 40, 1143-1150.

(25) Rueff, J. P.; Shukla, A.; Kaprolat, A.; Krisch, M.; Lorenzen, M.; Sette, F.; Verbeni, R. Phys. Rev. B: Condens. Matter Mater. Phys. 2001, 63, 132409.

(26) Lin, J.-F.; Struzhkin, V. V.; Jacobsen, S. D.; Hu, M. Y.; Chow, P.; Kung, J.; Liu, H.; Mao, H.-k.; Hemley, R. J. Nature 2005, 436, 377-380.

(27) Bergmann, U.; Horne, C.; Collins, T.; Workman, J.; Cramer, S. Chem. Phys. Lett. 1999, 302, 119-124.

(28) Rueff, J.-P.; Journel, L.; Petit, P.-E.; Farges, F. Phys. Rev. B: Condens. Matter Mater. Phys. 2004, 69, 235107.

(29) de Groot, F.; Glatzel, P.; Bergmann, U.; vanAken, P.; Barrea, R.; Klemme, S.; Havecker, M.; Knop-Gericke, A.; Heijboer, W.; Weckhuysen, B. J. Phys. Chem. B 2005, 109, 20751-20762.

(30) Briois, V.; Cartier dit Moulin, C.; Sainctavit, P.; Brouder, C.; Flank, A.-M. J. Am. Chem. Soc. 1995, 117, 1019-1026.

(31) Cartier, C.; Thuéry, P.; Verdaguer, M.; Zarembowitch, J.; Michalowicz, A. J. Phys. (Paris) 1986, 47, (C8) 563-568.

(32) Westre, T. E.; Kennepohl, P.; DeWitt, J. G.; Hedman, B.; Hodgson, K. O.; Solomon, E. I. J. Am. Chem. Soc. 1997, 119, 6297-6314.

(33) Krause, M. O.; Oliver, J. H. J. Phys. Chem. Ref. Data 1979, 8, $329-338$.

(34) Hämäläinen, K.; Siddons, D. P.; Hastings, J. B.; Berman, L. E. Phys. Rev. Lett. 1991, 67, 2850.

(35) Glatzel, P.; Bergmann, U.; Yano, J.; Visser, H.; Robblee, J. H.; Gu, W.; de Groot, F. M. F.; Christou, G.; Pecoraro, V. L.; Cramer, S. P.; Yachandra, V. K. J. Am. Chem. Soc. 2004, 32, 9946-9959.

(36) Carra, P.; Fabrizio, M.; Thole, B. T. Phys. Rev. Lett. 1995, 74, 3700 .

(37) Suzuki, C.; Kawai, J.; Tanizawa, J.-y.; Adachi, H.; Kawasaki, S.; Takano, M.; Mukoyama, T. Chem. Phys. 1999, 241, 17-27.

(38) Rueff, J.-P.; Krisch, M.; Cai, Y. Q.; Kaprolat, A.; Hanfland, M. Lorenzen, M.; Masciovecchio, C.; Verbeni, R.; Sette, F. Phys. Rev. B: Condens. Matter Mater. Phys. 1999, 60, 14510.

(39) Badro, J.; Rueff, J.-P.; Vankó, G.; Monaco, G.; Fiquet, G.; Guyot, F. Science 2004, 305, 383-386.

(40) Vankó, G.; Rueff, J.-P.; Mattila, A.; Németh, Z.; Shukla, A. Phys. Rev. B: Condens. Matter Mater. Phys. 2006, 73, 024424.

(41) Yoo, C. S.; Maddox, B.; Klepeis, J.-H. P.; Iota, V.; Evans, W.; McMahan, A.; Hu, M. Y.; Chow, P.; Somayazulu, M.; Hausermann, D.; Scalettar, R. T.; Pickett, W. E. Phys. Rev. Lett. 2005, 94, 115502. 